Rapid clinical response to adjuvant corticosteroids in chronic disseminated candidiasis complicated by granulomas and persistent fever in acute leukemia patients.

Fox $T A^{1,2}$, Halsey $R^{1}$, Pomplun $S^{l}$, Gant $V^{1,2}$, Grandage $V^{l}$, Mansour $M R^{1,2}$, Hough $R^{1,2}$, Khwaja $A^{1,2}$.

1 University College London Hospitals NHS Foundation Trust, London, UK

2 University College London, London, UK

Corresponding author: TA Fox, e-mail: t.fox @ucl.ac.uk 


\section{Rapid clinical response to adjuvant corticosteroids in chronic disseminated candidiasis complicated by granulomas and persistent fever in acute leukemia patients.}

We report successful resolution, using adjuvant corticosteroids, of a challenging clinical scenario in patients undergoing treatment for acute leukemia who developed chronic disseminated candidiasis (CDC) complicated by granuloma formation and immune reconstitution inflammatory syndrome (IRIS). The use of corticosteroids in the management of invasive fungal infections (IFIs) such as CDC is controversial. In this context, corticosteroids were used successfully to disrupt the granulomas and ameliorate the inflammatory response. Our results suggest that the addition of corticosteroids to antifungal agents is safe and should be considered as a treatment option in the difficult clinical contexts described.

Modern therapeutic approaches have led to an increasing number of patients who recover from a state of acquired immunodeficiency which may be complicated by an IRIS. IRIS is recognized as a complication of invasive fungal infections (IFIs) in patients undergoing intensive chemotherapy, presenting as persistence of symptoms or clinical deterioration following immunological recovery. Failure to recognize IRIS can have dire consequences for the hematology patient, as prolonged antimicrobial and antifungal therapy rather than anti-inflammatory drugs results in delays to treatment, morbidity and even mortality [1].

IFIs can cause significant morbidity and mortality in acute leukemia patients [2]. Prophylaxis has been variably adopted but breakthrough infections still occur in $3-17 \%$ of patients and IFIs have direct infection-related mortality in $25 \%$ of those affected [3]. 
An alternative, severe and disseminated clinical variant of IFI is recognized in $<5 \%$ of acute leukemia patients, can occur [4]. This is considered to consist of two distinct stages, namely the first, in which fungal dissemination occurs whilst the patient is in aplasia, and a second phase during neutrophil recovery, characterized by recurring fevers and hepatalgia in the absence of evidence of ongoing fungal infection. This second phase is increasingly thought to be the manifestation of an IRIS [1,5].

The use of corticosteroids in the treatment of acute IFIs is controversial, appears counterintuitive and they are not recommended in treatment guidelines [6,7]. Whilst numbers reported in the literature are low, corticosteroids appear to be critical to clinical improvement in IFI patients who have developed IRIS (see table 1[5,8-14]). Here, we describe a series of patients undergoing treatment for acute leukemia who developed the variant of disseminated IFI described above. Histology in all cases revealed fungal foci surrounded by extensive granulomas. All patients had prolonged and persistent symptoms despite anti-fungal therapy, attributed to IRIS. Corticosteroids were used successfully in all patients with rapid resolution of symptoms and normalization of inflammatory markers.

A retrospective case review was conducted of patients at University College London Hospital, a large tertiary haemato-oncology unit. Three patients were identified whose anti-leukemic treatment had been complicated by invasive fungal infection and IRIS who subsequently received steroids. All patients initially presented with febrile episodes during aplasia and were managed with empirical antibiotic and antifungal therapy as per local policy. Details of diagnosis, chemotherapy and fungal prophylaxis are shown in table 2. 
Patient one developed fevers 1 week into ALL induction chemotherapy and was managed with empirical antibiotics. Candida albicans was isolated in blood cultures and antifungal drugs were commenced (table 2). Liver lesions were identified on imaging and trans jugular liver biopsy was performed (figure 1). Histopathological examination revealed granuloma formation with central necrosis and palisaded histiocytes. Following diagnosis of IRIS, prednisolone was started (table 2). Following diagnosis of IRIS, prednisolone 10mg twice daily commenced as an outpatient which was then tapered over 16 weeks but stopped at 12 weeks prematurely due to patient compliance. Fevers settled 14 days after starting steroids. Chemotherapy was delayed and on cessation of fevers (some 12 weeks later) the patient proceeded with interim maintenance treatment and delayed intensification as part of the UKALL2011 protocol. Maintenance therapy with vincristine, 6-mercaptopurine and methotrexate was completed 2.5 years later with fluconazole prophylaxis given throughout. The patient remains in remission 8 months later.

Patient two received induction chemotherapy with FLA-IDA (fludarabine, cytarabine and idarubicin). A diagnosis of IFI was made in the context of persistent fevers and lung nodules on CT. MRI brain scan showed ring enhancing lesions, presumed of fungal etiology. (figure 1) Following neutrophil recovery, there were persistent high-grade fevers, repeat brain MRI showed progression of the intracerebral lesions, and multiple lesions were seen in the spleen and liver on ultrasound. Splenic biopsy showed multiple necrotizing granulomas which were negative on Diastase periodic acid Schiff staining but positive by PCR for Candida albicans. A diagnosis of IRIS secondary to IFI was made 8 weeks into treatment and dexamethasone was commenced (see table 2). Dexamethasone 
10mg daily was started initially with a dramatic response and resolution of fevers and improvement in inflammatory markers. On weaning/cessation of steroids, the fevers returned, and inflammatory markers rebounded. Dexamethasone was restarted at 3mg/day to good effect, but fevers recurred following conversion to prednisolone and a further reduction in dose. The patient was maintained on low dose prednisolone until treatment was consolidated with an allogeneic stem cell transplant. Antifungal and steroid therapy was continued up to and throughout the peri transplant period. The steroids were stopped 21 days after engraftment and the voriconazole ceased 7 months post-transplant. The patient remains in remission 20 months post-transplant.

Patient three achieved complete remission of AML following induction chemotherapy with FLAG-IDA and gemtuzumab and developed a presumed pulmonary IFI during consolidation therapy with CPX-351 (liposomal daunorubicin and cytarabine). High fevers persisted following neutrophil recovery, abdominal ultrasound showed multiple splenic nodules and biopsy showed necrotizing granulomata. (supplementary figure 2). Investigations for mycobacterial and fungal infection were negative and IRIS was diagnosed. Prednisolone 30mg/day was commenced with rapid resolution of fevers and improvement in inflammatory markers - steroids were successfully weaned over a period of 4 weeks (table 2). The patient underwent an allogeneic stem cell transplant four months later. Posaconazole was continued throughout the peri transplant period and stopped 9 weeks after engraftment. The patient remains in remission 18 months later.

The understanding of the pathophysiology of the IRIS associated with IFIs is incomplete. The described second stage following neutrophil recovery, accompanied by a 'paradoxical' clinical deterioration is thought to be due to the production of pro- 
inflammatory Th1 and Th17 responses [5,9]. Unregulated Th1/Th17 responses lead to increased interferon- $\gamma$ production, activating macrophages which promote granuloma formation and this process continues in a self-propagating loop [1].

Anti-inflammatory therapy with corticosteroids led to rapid resolution of symptoms and clinical improvement, permitting the consolidation of the patients' anti-leukemic treatment with further chemotherapy or allogeneic stem cell transplantation. Comparisons can be made with the successful use of corticosteroids to treat obstructive granulomata and infections in inherited chronic granulomatous disease (CGD) $[15,16]$. Our results are consistent with previous reports in the literature (table 1) which support the use of corticosteroids to treat IRIS in hematology patients.

Our case series is the first to demonstrate extensive granulomata surrounding fungal lesions in patients recovering from chemotherapy. We hypothesize that corticosteroids may aid granulomata disruption and penetration of anti-fungal agents. Our results, and, those of others, suggest that the addition of corticosteroids to antifungal agents is safe and should be considered as a treatment option in the difficult clinical contexts described.

\section{Acknowledgments}

TAF, AK and RH collected and analyzed the data and wrote the paper. RH provided and annotated the radiology images. SP provided and annotated the histopathology images. VG, MM, VG were involved in the clinical care of the patients and review of the final manuscript. The authors have no conflicts of interests to declare. VGant is grateful for support from the UCLH BRC. 


\section{References}

1. Delliere S, Guery R, Candon S, et al. Understanding Pathogenesis and Care Challenges of Immune Reconstitution Inflammatory Syndrome in Fungal Infections. J Fungi (Basel). 2018 Dec 17;4(4).

2. O'Reilly MA, Govender D, Kirkwood AA, et al. The incidence of invasive fungal infections in children, adolescents and young adults with acute lymphoblastic leukaemia/lymphoma treated with the UKALL2011 protocol: a multicentre retrospective study. Br J Haematol. 2019 Jul;186(2):327-329.

3. Koehler P, Hamprecht A, Bader O, et al. Epidemiology of invasive aspergillosis and azole resistance in patients with acute leukaemia: the SEPIA Study. Int J Antimicrob Agents. 2017 Feb;49(2):218-223.

4. Sallah S, Semelka RC, Wehbie R, et al. Hepatosplenic candidiasis in patients with acute leukaemia. Br J Haematol. 1999 Sep;106(3):697-701.

5. Chaussade H, Bastides F, Lissandre S, et al. Usefulness of corticosteroid therapy during chronic disseminated candidiasis: case reports and literature review. J Antimicrob Chemother. 2012 Jun;67(6):1493-5.

6. Lionakis MS, Kontoyiannis DP. Glucocorticoids and invasive fungal infections. Lancet. 2003 Nov 29;362(9398):1828-38.

7. Limper $\mathrm{AH}$. The changing spectrum of fungal infections in pulmonary and critical care practice: clinical approach to diagnosis. Proc Am Thorac Soc. 2010 May;7(3):163-8.

8. Conter C, Dupont B, Thiesse P, et al. Persistent fever and hepatosplenic candidiasis, efficiency of a corticoid therapy. J Mycol Med. 2007 Sep;17(3):194197.

9. Legrand F, Lecuit M, Dupont B, et al. Adjuvant corticosteroid therapy for chronic disseminated candidiasis. Clin Infect Dis. 2008 Mar 1;46(5):696-702.

10. Saint-Faust M, Boyer C, Gari-Toussaint M, et al. Adjuvant Corticosteroid Therapy in 2 Children With Hepatosplenic Candidiasis-related IRIS. J Pediat Hematol Onc. 2009 Oct;31(10):794-796.

11. Chandesris MO, Kelaidi C, Mechai F, et al. Granulocyte Colony Stimulating Factor-induced Exacerbation of Fungus-related Immune Restoration Inflammatory Syndrome: A Case of Chronic Disseminated Candidiasis Exacerbation. J Microbiol Immunol. 2010 Aug;43(4):339-343.

12. Bayram C, Fettah A, Yarali N, et al. Adjuvant corticosteroid therapy in hepatosplenic candidiasis-related iris. Mediterr J Hematol Infect Dis. 2012;4(1):e2012018.

13. Zajac-Spychala O, Ukielska B, Jonczyk-Potoczna K, et al. Chronic Disseminated Candidiasis Complicated by Immune Reconstitution Inflammatory Syndrome in Child with Acute Lymphoblastic Leukemia. Case Rep Hematol. 2016;2016:5960150.

14. Shkalim-Zemer V, Levi I, Fischer S, et al. Response of Symptomatic Persistent Chronic Disseminated Candidiasis to Corticosteroid Therapy in Immunosuppressed Pediatric Patients: Case Study and Review of the Literature. Pediatr Infect Dis J. 2018 Jul;37(7):686-690. 
15. Yamazaki-Nakashimada MA, Stiehm ER, Pietropaolo-Cienfuegos D, et al. Corticosteroid therapy for refractory infections in chronic granulomatous disease: case reports and review of the literature. Ann Allergy Asthma Immunol. 2006 Aug;97(2):257-61.

16. Magnani A, Brosselin P, Beaute J, et al. Inflammatory manifestations in a single-center cohort of patients with chronic granulomatous disease. J Allergy Clin Immunol. 2014 Sep;134(3):655-662 e8. 